Review Article

\title{
The Effects of Yoga on Patients with Parkinson's Disease: A Meta-Analysis of Randomized Controlled Trials
}

\author{
Mengke Ban, ${ }^{1}$ Xuejing Yue $\mathbb{D}^{2},{ }^{2}$ Pengyu Dou, ${ }^{1}$ and Ping Zhang $\mathbb{D}^{1}$ \\ ${ }^{1}$ Department of Neurology, The First Affiliated Hospital of Xinxiang Medical University, Xinxiang, China \\ ${ }^{2}$ Xinxiang Medical University, Xinxiang, China \\ Correspondence should be addressed to Xuejing Yue; jing04001825@126.com and Ping Zhang; zhangpingsjnk@163.com
}

Received 18 February 2021; Revised 19 April 2021; Accepted 26 May 2021; Published 5 July 2021

Academic Editor: Karsten Witt

Copyright (c) 2021 Mengke Ban et al. This is an open access article distributed under the Creative Commons Attribution License, which permits unrestricted use, distribution, and reproduction in any medium, provided the original work is properly cited.

\begin{abstract}
Background and Purpose. The manifestations of motor and nonmotor dysfunctions in Parkinson's disease (PD), which are intimately connected, have been shown to reduce quality of life (QoL). It has been demonstrated that yoga could benefit PD patients. However, there was no consensus on the impact of yoga on PD. This meta-analysis is aimed at investigating the effects of yoga intervention on motor function, nonmotor function, and QoL in patients with PD. Methods. A meta-analysis was conducted by systematically searching PubMed, Embase, and Cochrane Library databases till August 2020 for studies published in English. The reference lists of eligible studies were also searched. The motor symptoms (UPDRS-Part III), balance function (BBS and BESTest), functional mobility (TUG), anxiety (HADS and BAI), depression (HADS and BDI), and the quality of life (PDQ-39 and PDQ-8) were the primary evaluation indexes. Results. Ten studies including 359 participants were included in this meta-analysis. The pooled results showed significant difference between the yoga training group and the control group. Patients in the yoga training group had better functional outcomes in terms of motor status ( $\mathrm{MD}=-5.64 ; 95 \% \mathrm{CI},-8.57$ to -2.7$)$, balance function ( $\mathrm{SMD}=0.42 ; 95 \% \mathrm{CI}, 0.08$ to 0.77$)$, functional mobility $(\mathrm{MD}=-1.71 ; 95 \% \mathrm{CI},-2.58$ to -0.84$)$, anxiety scale scores $(\mathrm{SMD}=-0.72 ; 95 \% \mathrm{CI},-1.01$ to -0.43$)$, depression scale scores $(\mathrm{SMD}=-0.92 ; 95 \% \mathrm{CI},-1.22$ to -0.62$)$, and $\mathrm{QoL}(\mathrm{SMD}=-0.54$; $95 \% \mathrm{CI},-0.97$ to -0.11$)$. Conclusion. Our pooled results showed the benefits of yoga in improving motor function, balance, functional mobility, reducing anxiety and depression, and increasing QoL in PD patients.
\end{abstract}

\section{Introduction}

$\mathrm{PD}$ is the second most prevalent neurodegenerative disease worldwide, only behind Alzheimer's disease [1]. It primarily occurs in middle-aged and elderly people, influencing about $1 \%$ of the population above the age of 60 . The clinical manifestations associated with PD consist of motor symptoms and nonmotor symptoms. Bradykinesia, rigidity, static tremor, and postural instability are typical motor symptoms [2], which in turn affect the functional mobility, balance, and gait of patients, as well as increase the risk of falling. Anxiety, depression, and cognitive impairment are pervasive nonmotor symptoms [3], which subsequently have an immense impact on QoL.

As a chronic progressive disease, $\mathrm{PD}$ is mainly treated with drugs combined with routine rehabilitation training. The main purpose of the treatment is to slow down the progression of the disease, reduce the clinical symptoms, and improve QoL in PD patients. However, long-term medication may lead to several motor complications [4]. Previous studies have revealed that rehabilitation therapy, as an adjuvant strategy, could slow down the progression of PD and improve motor function, functional mobility, balance, and the health-related QoL of the patients. Holistic interventions that address both motor and nonmotor symptoms of PD simultaneously are gaining popularity. In 2002, the National Center for Complementary and Alternative Medicine of the United States conducted a survey on adults aged over 18 years, which revealed that yoga had become the fifth most frequently used method of rehabilitation therapy [5]. Yoga, a method of physical and psychological exercise and rehabilitation, has been widely used worldwide. It consists of postures (asanas), breathing (pranayama), and meditation (dhyana). Some studies have suggested that yoga can produce great benefits in ameliorating both motor and nonmotor symptoms of PD patients. 
Although recent studies have reviewed the efficacy of yoga in the treatment of PD [6-8], a comprehensive view with regard to its therapeutic effects has not been reported. Some randomized clinical trials (RCTs) have evaluated the impact of yoga on motor as well as nonmotor function in PD, but the results were controversial. Ni et al. [9] and Bega and Stein [10] used Unified Parkinson's Disease Rating Scale (UPDRS), Berg Balance Scale (BBS), and Timed "Up \& Go" (TUG) test to evaluate the motor functions of PD patients, and significant improvement was observed after yoga exercise. Bega and Stein [10], Kwok et al. [11], and Boulgarides et al. [12] mentioned the benefits of yoga on anxiety. However, the study conducted by Myers et al. [13] showed no positive results. To investigate whether yoga was beneficial to PD patients, and in what ways it could ameliorate the symptoms of patients and improve their quality of life, a meta-analysis of published RCTs on the effects of yoga in the treatment of $\mathrm{PD}$, including motor functions, balance, mobility, anxiety, depression, and QoL, was conducted.

\section{Methods}

2.1. Literature Search. Two reviewers (Ping Zhang and Mengke Ban) independently searched PubMed, Embase, and Cochrane Library databases up to August 2020 with the following search terms: "Parkinson's disease" and "yoga." The complete search strategy was described in the appendix. In addition, the list of references in all eligible articles was also reviewed to identify other relevant articles. Any disagreements during the literature search process were addressed by discussion with a third reviewer (Xuejing Yue).

2.2. Study Eligibility. The inclusion criteria were as follows: (1) studies designed as RCTs; (2) participants diagnosed with PD (according to the standard of MDS-UPDRS method), rather than Parkinson's syndrome; (3) participants should be divided into at least two groups, the experimental group with yoga intervention and the control group (i.e., resistance training, proprioceptive training, conventional balance exercise, waiting-list, routine care, and nonexercise control); and (4) the study should report at least one preestablished outcome. Studies were excluded if they met the following criteria: (1) case reports, conference abstracts, reviews articles, unrelated studies, comments, and cross-sectional studies; (2) duplicate articles and studies lacking relevant outcomes; and (3) studies published in non-English language.

2.3. Quality Assessment. To assess the quality of the included studies, the researchers independently evaluated the risk bias using Review Manager 5.4, which involved seven domains of bias: random sequence generation (selection bias), allocation concealment (selection bias), blinding of participants and personnel (performance bias), blinding of outcome assessment (detection bias), incomplete outcome data (attrition bias), selective reporting (reporting bias), and other sources of bias (other bias). Each domain was judged as one of these three risk categories: low risk of bias, unclear risk of bias, and high risk of bias. If there was considerable controversy during the abovementioned processes, a consensus was reached by discussion with a third reviewer (Xuejing Yue).

2.4. Data and Variables Extraction. The following baseline data from the included literature were extracted: first author and publication year, study design, study region, characteristics of participants, experimental intervention, control intervention, and outcome measures. The outcome variables of yoga and control groups collected included the mean and standard deviation of prepost changes of UPDRS-III scores, BBS scores, TUG scores, BEST scores, HADS scores, BAI scores, BDI scores, and PDQ scores. The Unified Parkinson's Disease Rating Scale-Part III (UPDRS-Part III) was used to evaluate the motor function, which ranged from 0 to 44 , with higher scores indicating worse parkinsonism. The balance was assessed by BBS and Mini-Balance Evaluation Systems Test (Mini-BESTest). The former included 14 items with a total score of 56, and the latter contained 14 motor functional tests, with the higher scores indicating higher level of balance. The TUG measured functional mobility, and the lower time indicated better mobility. The Hospital Anxiety and Depression Scale (HADS) was used to assess the symptoms of anxiety or depression, and a high score was associated with high level of psychological pain. Anxiety and depression were assessed by Beck Anxiety Inventory (BAI) and Beck Depression Inventory (BDI), with higher scores indicating the more severe symptoms. The Parkinson's Disease Questionnaire-39 (PDQ-39) and the Parkinson's Disease Questionnaire-8 (PDQ-8) were validated tools used for assessing QoL, and the lower scores indicated an improved QoL.

2.5. Statistical Analysis. For continuous data, the mean differences (MDs) or standardized mean differences (SMDs), with 95\% confidence intervals (CIs), were calculated using RevMan 5.4. If the mean or standard deviation of the changes was not provided, then Cochrane software system was used for the calculation of MD or SMD. Besides, chi-square test and Higgins $I^{2}$ statistics were used to assess the heterogeneity across studies. An $I^{2}$ value of $50 \%$ or higher was considered an indicator of substantial heterogeneity, and a randomeffects model would be adopted. Meanwhile, subgroup analysis was implemented to explore the potential sources of heterogeneity. The fixed-effects model was used when the $I^{2}$ value was less than $50 \%$, indicating that the heterogeneity among the studies was not significant. All reported probability $(P)$ values were two-sided, and $P \leq 0.05$ was considered statistically significant.

\section{Results}

3.1. Search Results. Figure 1 summarized the process of literature search. A total of 62 relevant documents were identified from PubMed, Embase, and Cochrane Library databases, and 35 non-RCT articles were removed. Basing on the PICO format, the title and abstracts of these documents were carefully screened; then, 16 articles which met the eligibility criteria were selected. After reviewing the full text, 2 studies published by Sharma et al. [14] were found to come from the same trial, with each study containing different outcome 


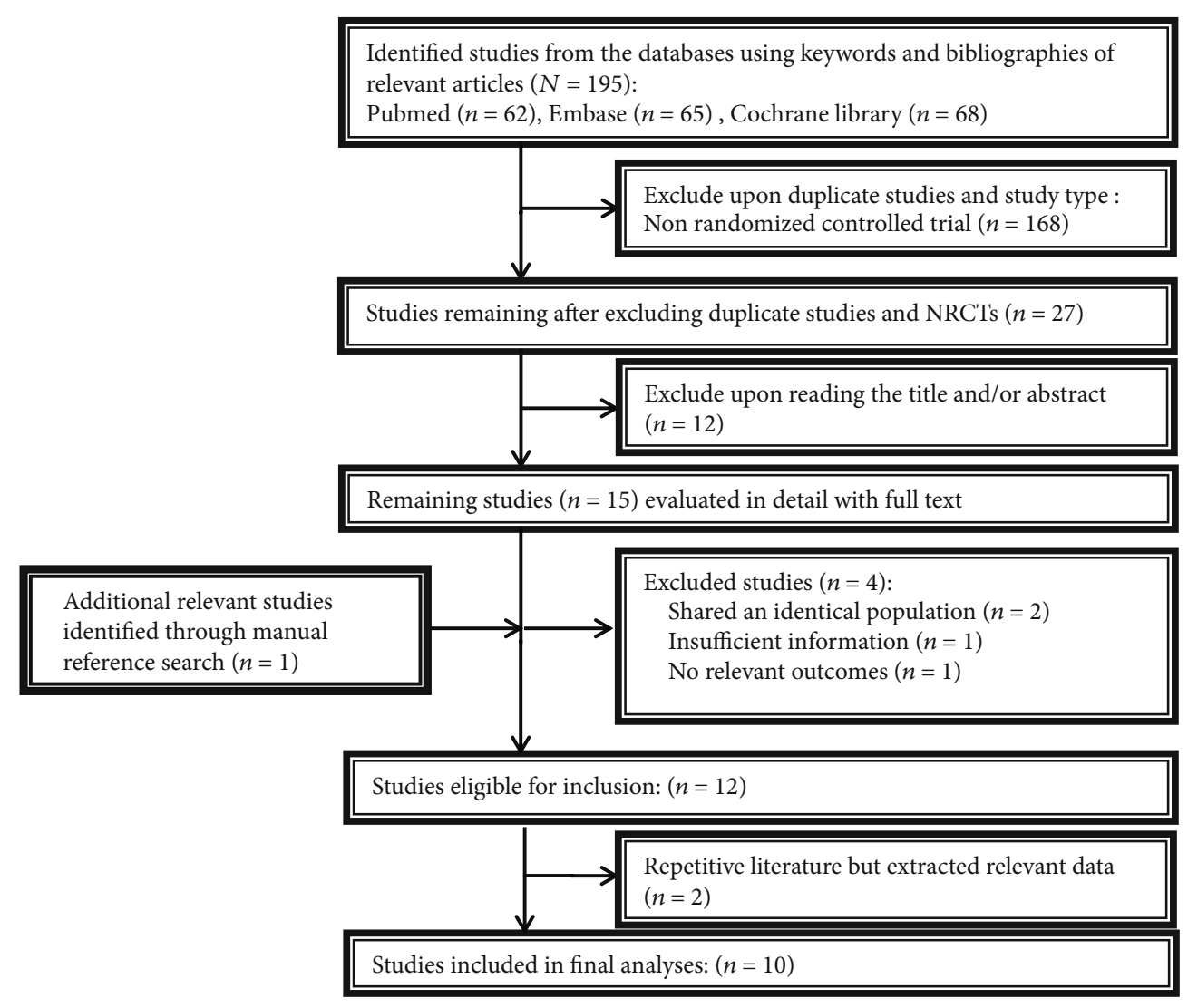

Figure 1: Flowchart of study selection.

measures. The article was excluded because there were no corresponding outcome measures, and the one by Colgrove in 2012 was retained. Kwok et al. [11, 15] have published 2 articles from the same trial. The publication in 2017 [15] was excluded because it was a study protocol, while the paper published in 2019 [11] was classified as eligible. Ni et al. $[9,16]$ reported 2 repetitive articles originated from the same trial, and both studies had their own unique outcome indicators. Our meta-analysis extracted different outcome variables from these two articles and regarded them as the same study. Hawkins et al., Van Puymbroeck et al., and Walter et al. [17-19] published 3 articles for the same trial, and a mixed study was excluded, and the outcome variables needed were extracted from the remaining 2 articles $[18,19]$, which were also regarded as the same study. Finally, a total of 10 studies were included.

3.2. Study Characteristics. A total of 359 participants were included in our meta-analysis. All subjects in the intervention and the control group were diagnosed with $\mathrm{PD}$, with a Hoehn \& Yahr scores ranging from 1 to 3 . The average age of the patients ranged from 60 to 80 years. Furthermore, most of the studies were conducted in America. All studies were compared between the trial group (yoga exercise) and the control group (i.e., resistance training, proprioceptive training, conventional balance exercise, waiting-list, routine care, and nonexercise control). The baseline characteristics of all patients were summarized in Table 1.
3.3. Quality Assessment. A total of 10 RCTs evaluated the risk of bias, and 8 trials (80\%) generated sufficient randomization sequence and detailed information about randomization. Allocation concealment was mentioned in 3 trials (30\%). It was not possible to blind the participants to group allocation, but the system evaluator's judgment of outcome was almost unlikely to be affected by the lack of blind method. Therefore, performance bias was unavoidable in all trials. In addition, 8 trials (80\%) reported the blinding of outcome assessment, and 7 trials (70\%) provided complete data for the outcomes. Regarding selective reporting bias, only 2 trials (20\%) met the requirements. The overall quality of included studies was medium to low (Figure 2).

\subsection{Outcomes}

3.4.1. Motor Symptoms. Seven studies [9-12, 18, 21, 22] with 255 patients assessed motor status by UPDRS-III. A significant improvement in UPDRS-III scores was observed among the patients who performed yoga when compared to those in the control group $(\mathrm{MD}=-5.64 ; 95 \% \mathrm{CI},-8.57$ to -2.7 ; $P=0.0002) . I^{2}$ analyses revealed significant heterogeneity $\left(I^{2}=64 \% ; P=0.01\right)$, so random-effects model was used. According to sensitivity analysis, $I^{2}$ was reduced to $0 \%$ when the article conducted by $\mathrm{Ni}$ et al. [9] was omitted (Figure 3). Subgroup analysis showed that age was the source of significant heterogeneity (Figure 4). 
TABLE 1: Characteristics of included studies.

\begin{tabular}{|c|c|c|c|c|c|c|}
\hline Study & Region & $\begin{array}{l}\text { Study } \\
\text { design }\end{array}$ & $\begin{array}{l}\text { Participants and group } \\
\text { allocation }\end{array}$ & Intervention & Control & $\begin{array}{c}\text { Main outcome } \\
\text { measures }\end{array}$ \\
\hline $\begin{array}{l}\text { Bega et al. } \\
\text { (2016) [10] }\end{array}$ & USA & RCT & $\begin{array}{l}\text { Experimental group }(n: 7 ;) \text { : } \\
\text { age (y): } 67.9 \pm 10.9 ; \text { gender } \\
\text { (M/F): } 5 / 2 ; \text { HY: } 2.3 \pm 0.4 \\
\text { Control group }(n: 7): \text { age } \\
\text { (y): } 66.7 \pm 9.3 \text {; gender } \\
(\mathrm{M} / \mathrm{F}): 6 / 1 ; \mathrm{HY}: 2.4 \pm 0.5\end{array}$ & $\begin{array}{l}\text { Experimental group (yoga): yoga } \\
\text { exercise } 60 \text { min, } 2 / \text { wk } \times 12 \mathrm{wk} \\
\text { (deep breathing exercises and } \\
\text { relaxation techniques; pose } \\
\text { targeting; meditation) }\end{array}$ & $\begin{array}{l}\text { Control group: } \\
\text { resistance training }\end{array}$ & $\begin{array}{c}\text { TUG, UPDRS, } \\
\text { BBS, PDQ-39, BDI }\end{array}$ \\
\hline $\begin{array}{l}\text { Boulgarides } \\
\text { et al. (2014) [12] }\end{array}$ & USA & $\mathrm{RCT}$ & $\begin{array}{c}\text { Experimental group }(n: \\
\text { 10): age }(y): 65.7 ; \text { gender } \\
\text { (M/F): 7/3; HY: } 2.6 \\
\text { Control group (PD): age } \\
\text { (y): 65.7; gender }(\mathrm{M} / \mathrm{F}): \\
\text { 7/3; HY: } 2.6\end{array}$ & $\begin{array}{l}\text { Experimental group (yoga): yoga } \\
\text { exercise } 60 \text { min, } 1 / \text { wk } \times 8 \mathrm{wk} \\
\text { (postural training; meditation; } \\
\text { deep relaxation) }\end{array}$ & $\begin{array}{l}\text { Control group: } \\
\text { wait-list }\end{array}$ & $\begin{array}{l}\text { UPDRS, HADS, } \\
\text { BBS }\end{array}$ \\
\hline $\begin{array}{l}\text { Cherup et al. } \\
(2020) \text { [20] }\end{array}$ & USA & $\mathrm{RCT}$ & $\begin{array}{l}\text { Experimental group }(n: \\
\text { 15): age }(\mathrm{y}): 69.8 \pm 7.3 ; \\
\text { gender }(\mathrm{M} / \mathrm{F}): 10 / 5 \\
\text { Control group }(n: 18): \text { age } \\
\text { (y): } 71.4 \pm 12.1 ; \text { gender } \\
(\mathrm{M} / \mathrm{F}): 11 / 7\end{array}$ & $\begin{array}{l}\text { Experimental group (yoga): yoga } \\
\text { exercise } 45 \mathrm{~min}, 2 / \mathrm{wk} \times 12 \mathrm{wk} \\
\text { (postural training; meditation) }\end{array}$ & $\begin{array}{l}\text { Control group: } \\
\text { proprioceptive } \\
\text { training } 45 \mathrm{~min}, 2 \\
/ \mathrm{wk} \times 12 \mathrm{wk}\end{array}$ & TUG \\
\hline $\begin{array}{l}\text { Cheung et al. } \\
\text { (2018) [21] }\end{array}$ & Australia & $\mathrm{RCT}$ & $\begin{array}{l}\text { Experimental group }(n: \\
\text { 10): age }(\mathrm{y}): 63.5 \pm 8.5 ; \\
\text { gender (M/F): } 7 / 6 ; \text { HY: } 2 \\
\quad \pm 0.8 \\
\text { Control group }(n: 10): \text { age } \\
\text { (y): } 65.8 \pm 6.6 ; \text { gender } \\
(\mathrm{M} / \mathrm{F}): 8 / 5 ; \mathrm{HY}: 2 \pm 0.8\end{array}$ & $\begin{array}{l}\text { Experimental group (yoga): yoga } \\
\text { exercise } 60 \text { min, } 2 / \text { wk } \times 12 \mathrm{wk} \\
\text { (poses; breathing techniques; } \\
\text { meditation) }\end{array}$ & $\begin{array}{l}\text { Control group: the } \\
\text { wait-list group: } \\
\text { usual care }\end{array}$ & $\begin{array}{l}\text { UPDRS, MoCA, } \\
\text { Beck Depression } \\
\text { Inventory }\end{array}$ \\
\hline $\begin{array}{l}\text { Colgrove et al. } \\
(2012)[22]\end{array}$ & USA & $\mathrm{RCT}$ & $\begin{array}{c}\text { Experimental group }(n: 8) \text { : } \\
\text { age }(\mathrm{y}): 62.8 \pm 13.2 \text {; gender } \\
(\mathrm{M} / \mathrm{F}): 2 / 6 ; \mathrm{HY}: 1.3 \pm 0.3 ; \\
\text { duration of disease }(\mathrm{y}): 3.8 \\
\pm 2.9 \\
\text { Control group }(n: 5): \text { age } \\
(\mathrm{y}): 73.4 \pm 6.5 ; \text { gender } \\
(\mathrm{M} / \mathrm{F}): 4 / 1 ; \mathrm{HY}: 1.2 \pm 0.4 \\
\text { duration of disease }(\mathrm{y}): 3.7 \\
\pm 22.2\end{array}$ & $\begin{array}{l}\text { Experimental group (yoga): yoga } \\
\text { exercise } 60 \text { min, } 2 / \text { wk } \times 12 \mathrm{wk} \\
\text { (poses; breathing techniques; } \\
\text { meditation) }\end{array}$ & $\begin{array}{l}\text { Control group: } \\
\text { yoga sessions } 12 \\
\text { wk }\end{array}$ & $\begin{array}{l}\text { UPDRS-III, BBS, } \\
\text { ROM, muscle } \\
\text { strength gait } \\
\text { (postural sway and } \\
\text { gait initiation) }\end{array}$ \\
\hline $\begin{array}{l}\text { Jojo et al. (2019) } \\
\text { [11] }\end{array}$ & $\begin{array}{c}\text { China } \\
\text { (Hong Kong) }\end{array}$ & $\mathrm{RCT}$ & $\begin{array}{l}\text { Experimental group ( } n \text { : } \\
\text { 71): age (y): } 63.7 \pm 8.2 ; \\
\text { gender }(\mathrm{M} / \mathrm{F}): 37 / 34 \\
\text { Control group }(n: 67): \text { age } \\
\text { (y): } 63.5 \pm 9.3 ; \text { gender } \\
(\mathrm{M} / \mathrm{F}): 28 / 39\end{array}$ & $\begin{array}{l}\text { Experimental group (yoga): yoga } \\
\text { exercise } 90 \mathrm{~min} / \mathrm{wk} \times 8 \mathrm{wk} \\
\text { (breathing exercise }(15 \mathrm{~min}) \\
\text { mindfulness practice }(15 \mathrm{~min}) \\
\text { yoga practice }(60 \mathrm{~min}))\end{array}$ & $\begin{array}{l}\text { Control group: } \\
\text { stretching and } \\
\text { resistance } \\
\text { exercises } 60 \mathrm{~min} / \\
\text { wk } \times 8 \mathrm{wk}\end{array}$ & $\begin{array}{l}\text { HADS, UPDRS- } \\
\text { III, TUG, HWS, } \\
\text { PDQ-8 }\end{array}$ \\
\hline $\begin{array}{l}\text { Khuzema et al. } \\
\text { (2020) [23] }\end{array}$ & India & RCT & $\begin{array}{c}\text { Experimental group }(n: 9) \text { : } \\
\text { age }(\mathrm{y}): 68.11 \pm 4.23 ; \\
\text { gender }(\mathrm{M} / \mathrm{F}): 6 / 3 ; \\
\text { duration of disease }(\mathrm{y}): 6.2 \\
\pm 1.67 \\
\text { Control group }(n: 9): \text { age } \\
\text { (y): } 70.89 \pm 6.01 \text {; gender } \\
(\mathrm{M} / \mathrm{F}): 7 / 2 ; \text { duration of } \\
\text { disease }(\mathrm{y}): 5.23 \pm 3.12\end{array}$ & $\begin{array}{l}\text { Experimental group (yoga): yoga } \\
\text { exercise } 30-40 \text { min, } 8 \text { wk (postural } \\
\text { training; deep breathing exercises) }\end{array}$ & $\begin{array}{l}\text { Control group: } \\
\text { conventional } \\
\text { balance exercise } \\
30-40 \mathrm{~min}, 8 \mathrm{wk}\end{array}$ & $\begin{array}{l}\text { BBS, TUG, 10- } \\
\text { Minute Walk test }\end{array}$ \\
\hline $\begin{array}{l}\text { Myers et al. } \\
(2020)[13]\end{array}$ & USA & $\mathrm{RCT}$ & $\begin{array}{l}\text { Experimental group }(n: \\
\text { 13): age }(\mathrm{y}): 70.5 \pm 8.7 ; \\
\text { gender }(\mathrm{M} / \mathrm{F}): 7 / 6 ; \mathrm{HY}: 2 \\
\text { Control group ( } n: 13): \text { age } \\
\text { (y): } 65.0 \pm 8.7 ; \text { gender } \\
\text { (M/F): } 8 / 5 ; \text { HY: } 2\end{array}$ & $\begin{array}{l}\text { Experimental group (yoga): yoga } \\
\text { exercise } 60 \text { min, } 2 / \mathrm{wk} \times 12 \mathrm{wk} \\
\quad \text { (relaxation and meditation } 5 \\
\text { minutes; gentle spinal movements } \\
10 \text { minutes; standing poses } 30-35 \\
\text { minutes; cool down } 5-10 \text { minutes; } \\
\text { rest and relaxation } 5 \text { minutes) }\end{array}$ & $\begin{array}{l}\text { Control group: } \\
\text { usual daily } \\
\text { routines } 12 \mathrm{wk}\end{array}$ & BESTest, BAI \\
\hline
\end{tabular}


TABLE 1: Continued.

\begin{tabular}{|c|c|c|c|c|c|c|}
\hline Study & Region & $\begin{array}{l}\text { Study } \\
\text { design }\end{array}$ & $\begin{array}{l}\text { Participants and group } \\
\text { allocation }\end{array}$ & Intervention & Control & $\begin{array}{l}\text { Main outcome } \\
\text { measures }\end{array}$ \\
\hline $\begin{array}{l}\text { Ni et al. }(2016) \\
\text { (same study } \\
\text { with Ni et al. } \\
2016)[9,16]\end{array}$ & USA & RCT & $\begin{array}{c}\text { Experimental group }(n: \\
\text { 13): age }(\mathrm{y}): 71.2 \pm 6.5 ; \\
\text { gender (M/F): } 11 / 2 ; \mathrm{HY} \text { : } \\
2.2 \pm 0.7 \text {; duration of } \\
\text { disease }(\mathrm{y}): 6.9 \pm 6.3 \\
\text { Control group }(n: 10) \text { : age } \\
\text { (y): } 74.9 \pm 8.3 \text {; gender } \\
(\mathrm{M} / \mathrm{F}): 4 / 6 ; \mathrm{HY}: 2.1 \pm 0.7 \\
\text { duration of disease }(\mathrm{y}): 5.9 \\
\pm 6.2\end{array}$ & $\begin{array}{l}\text { Experimental group (yoga): yoga } \\
\text { exercise } 1 \mathrm{~h}, 2 / \text { wk } \times 12 \text { wk (poses } \\
\text { and deep breathing exercises) }\end{array}$ & $\begin{array}{l}\text { Control group: } 1 \text { - } \\
\text { hour nonexercise, } \\
\text { health education } \\
\text { class, } 1 / \mathrm{mo} \times 3 \mathrm{mo}\end{array}$ & $\begin{array}{l}\text { UPDRS-III, BBS, } \\
\text { Mini-BESTest, } \\
\text { TUG, PDQ-39 }\end{array}$ \\
\hline $\begin{array}{l}\text { Puymbroeck } \\
\text { et al. (2018) } \\
\text { (same study } \\
\text { with Walter } \\
\text { et al. 2019) [18, } \\
\text { 19] }\end{array}$ & USA & RCT & $\begin{array}{c}\text { Experimental group }(n \text { : } \\
\text { 15): age }(\mathrm{y}): 65.53 \pm 6.09 ; \\
\text { gender }(\mathrm{M} / \mathrm{F}): 10 / 5 \\
\text { Control group }(n: 12): \text { age } \\
\text { (y): } 70.5 \pm 4.44 \text {; gender } \\
(\mathrm{M} / \mathrm{F}): 7 / 5\end{array}$ & $\begin{array}{l}\text { Experimental group (yoga): yoga } \\
\text { exercise } 60 \mathrm{~min}, 2 / \mathrm{wk} \times 8 \mathrm{wk} \\
\text { (posture; pranayama; dhyana) }\end{array}$ & $\begin{array}{l}\text { Control group: } \\
\text { usual care } 8 \mathrm{wk}\end{array}$ & $\begin{array}{l}\text { UPDRS, Mini- } \\
\text { BESTest FGA, } \\
\text { FOG, PFS-16, } \\
\text { PDQ-8 }\end{array}$ \\
\hline
\end{tabular}

RCT: randomized controlled trial; M: male; F: female; values are mean \pm SD; HY: Hoehn and Yahr scale; min: minute; wk: week; mo: month; TUG: Timed Up and Go test; UPDRS: Unified Parkinson's Disease Rating Scale; UPDRS-III: the motor subscale of the Unified Parkinson's Disease Rating Scale; BBS: Berg Balance Scale; PDQ-39: Parkinson's Disease Questionnaire-39; BDI: Beck Depression Inventory; HADS: Hospital Anxiety and Depression Scale; MoCA: Montreal Cognitive Assessment; ROM: range-of-motion; HWS: Holistic Well-being Scale; PDQ-8: Parkinson's Disease Questionnaire-8; Mini-BESTest: Mini-Balance Evaluation Systems Test; BAI: Beck Anxiety Inventory; FGA: Functional Gait Assessment; FoG: Freezing of Gait Questionnaire; PFS-16: Parkinson's Fatigue Scale-16.

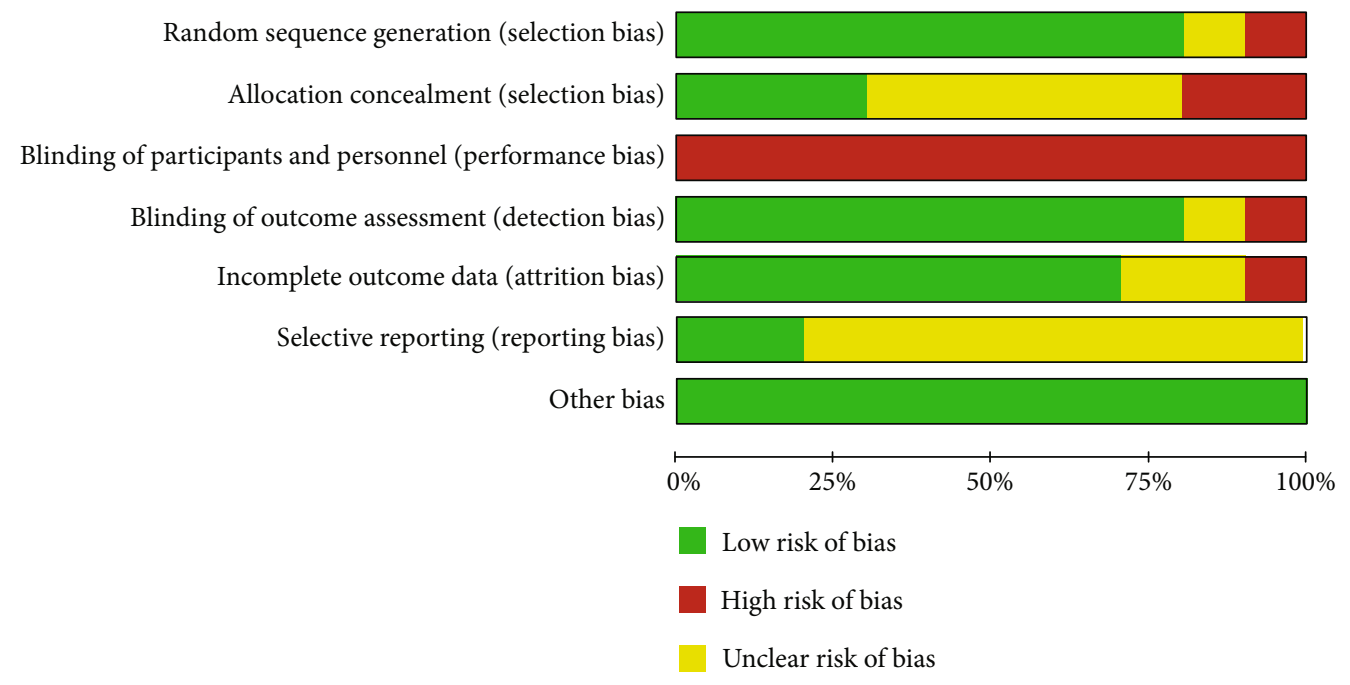

FIGURE 2: Graph of the risk of bias: percentages across all included studies.

3.4.2. Balance Function. Seven studies $[9,10,12,13,18,22$, 23 with 141 patients evaluated the balance function of $\mathrm{PD}$, among which 5 studies were assessed by BBS scores and 2 studies used the BESTest scale to evaluate the balance function. Compared with the control group, the pooled results showed that yoga intervention significantly promoted postural instability (SMD $=0.42 ; 95 \% \mathrm{CI}, 0.08$ to $0.77 ; P=0.02$ ). The heterogeneity test results of the included literatures showed no significance $\left(I^{2}=36 \% ; P=0.15\right)$ (Figure 5).

3.4.3. Functional Mobility. Five studies [9-11, 20, 23] involving 226 patients assessed the functional mobility by TUG. The results $(\mathrm{MD}=-1.71 ; 95 \% \mathrm{CI},-2.58$ to $-0.84 ; P=0.0001)$ demonstrated that yoga had a significant effect on improving the functional mobility of PD. There was no evidence of heterogeneity $\left(I^{2}=0 \% ; P=0.57\right)$ (Figure 6).

3.4.4. Anxiety Scale Scores. Four studies [10-13] covering 198 patients assessed nonmotor symptoms by anxiety scale scores. Among these, 2 studies used HADS and 2 studies used BAI to evaluate the efficacy of yoga on anxiety symptoms. The results showed that the scores were significantly decreased with yoga when compared to the control (SMD $=-0.72 ; 95 \% \mathrm{CI},-1.01$ to -0.43; $P<0.00001)$. There was no significant heterogeneity among the studies $\left(I^{2}=17 \% ; P=0.31\right)$ (Figure 7$)$.

3.4.5. Depression Scale Scores. Four studies [10-12, 21] covering 192 patients assessed nonmotor symptoms by depression scale scores. Two studies used HADS, and two used 


\begin{tabular}{lrrrrrrrr} 
& \multicolumn{3}{c}{ Yoga } & \multicolumn{4}{c}{ Control } & \multicolumn{2}{c}{ Mean Difference } \\
Study or subgroup & Mean & SD & Total & Mean & SD & Total & & IV, Random, 95\% CI \\
\hline Bega 2016 & -3.9 & 6.33 & 7 & -1.3 & 4.42 & 7 & $12.6 \%$ & $-2.60[-8.32,3.12]$ \\
Boulgarides 2014 & 0.9 & 6.77 & 10 & 0.4 & 7.8 & 10 & $11.3 \%$ & $0.50[-5.90,6.90]$ \\
Cheung 2018 & -8.6 & 5.63 & 10 & -1.9 & 5.96 & 10 & $14.0 \%$ & $-6.70[-11.78,-1.62]$ \\
Colgrove 2012 & 11.43 & 2.06 & 8 & 17.5 & 4.37 & 5 & $16.5 \%$ & $-6.07[-10.16,-1.98]$ \\
Jojo 2019 & -13.8 & 9.09 & 71 & -9.11 & 9.61 & 67 & $19.0 \%$ & $-4.69[-7.81,-1.57]$ \\
Ni 2016 & -10.9 & 5.13 & 13 & 0.4 & 2.52 & 10 & $18.8 \%$ & $-11.30[-14.50,-8.10]$ \\
Puymbroeck 2018 & -6.4 & 8.35 & 15 & -1.17 & 13.42 & 12 & $7.8 \%$ & $-5.23[-13.92,3.46]$ \\
Total (95\% CI) & & & 134 & & & 121 & $100.0 \%$ & $-5.64[-8.57,-2.70]$
\end{tabular}

Heterogeneity: $\mathrm{Tau}^{2}=9.25 ; \mathrm{Chi}^{2}=16.45, \mathrm{df}=6(P=0.01) ; I^{2}=64 \%$ Test for overall effect: $Z=3.77(P=0.0002)$

Figure 3: Forest plot from the meta-analysis of yoga on motor status.

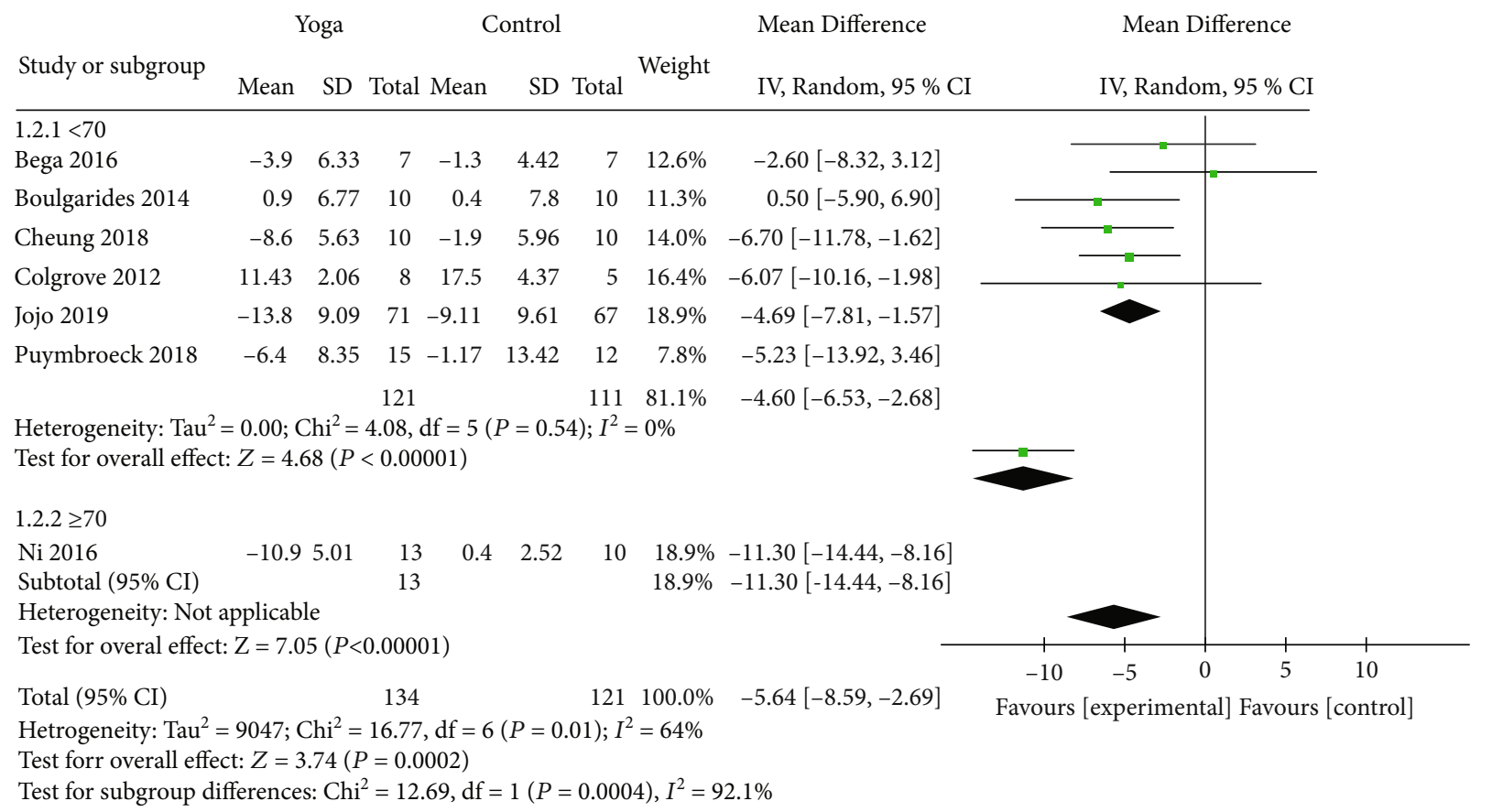

FIGURE 4: Subgroup analysis of differences among studies in UPDRS-III rating results.

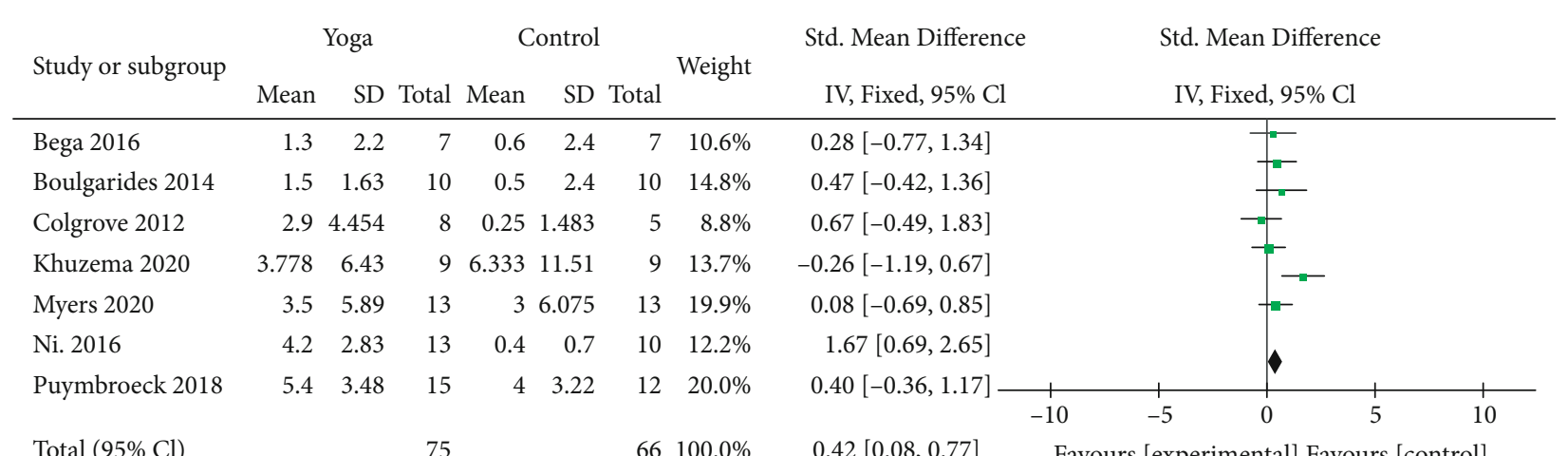

Heterogeneity: $\mathrm{Chi}^{2}=9.35, \mathrm{df}=6(P=0.15) ; I^{2}=36 \%$

Test for overall effect: $Z=2.42(P=0.02)$

Figure 5: Forest plot from the meta-analysis of yoga on balance function. 


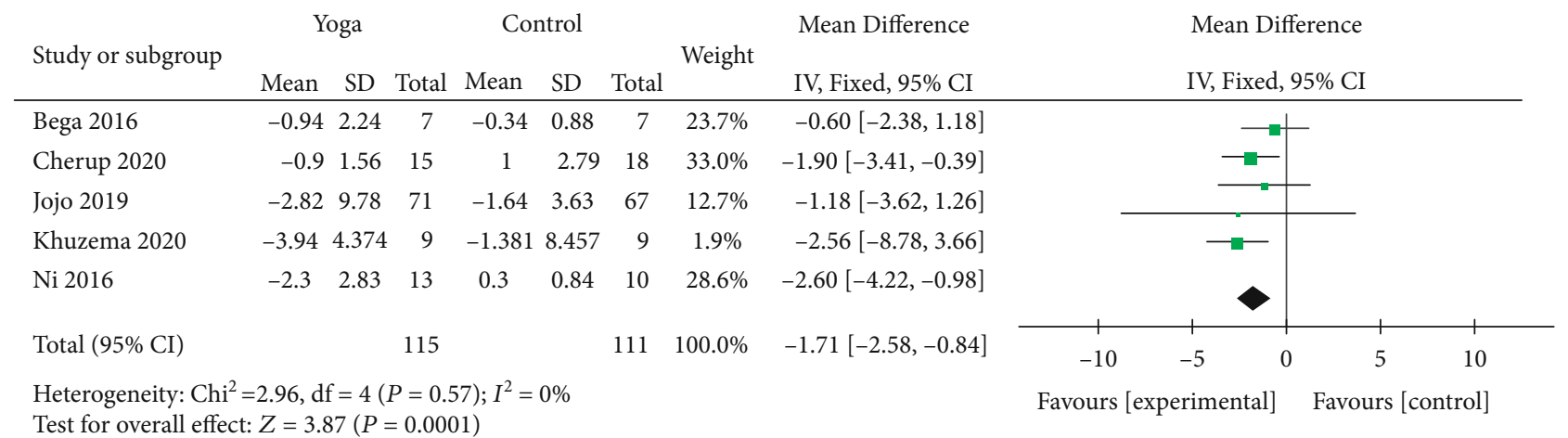

FIGURE 6: Forest plot from the meta-analysis of yoga on functional mobility.

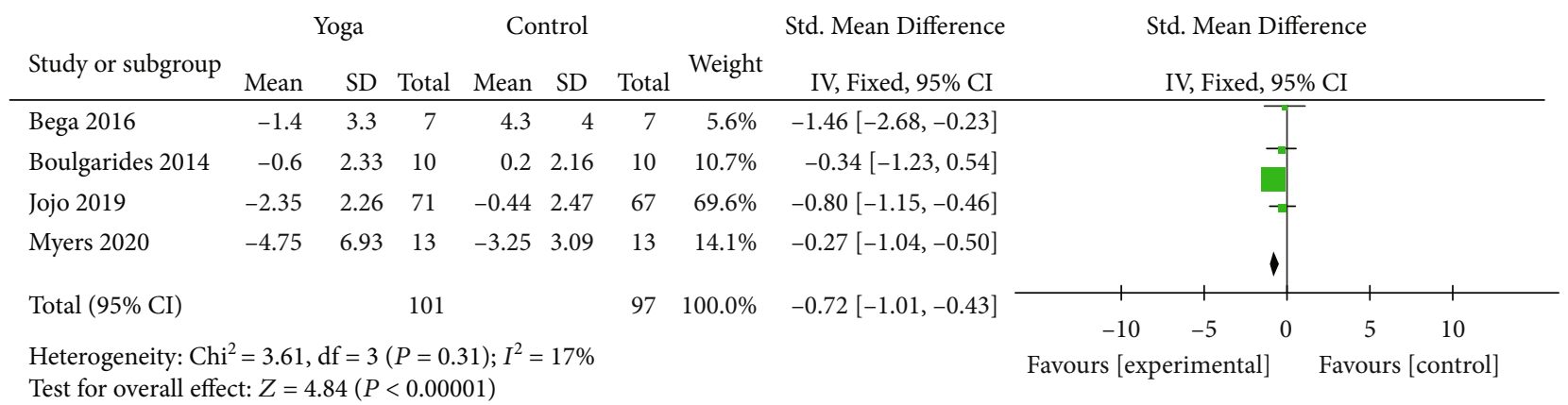

FiguRE 7: Forest plot from the meta-analysis of yoga on anxiety.

BDI to assess the depression symptom. The pooled results suggested a significant improvement in depression for patients in the yoga exercise group compared to the control group $(\mathrm{SMD}=-0.92 ; 95 \% \mathrm{CI},-1.22$ to $-0.62 P<0.00001)$. There was no significant heterogeneity $\left(I^{2}=0 \% ; P=0.44\right)$ (Figure 8 ).

3.4.6. Quality of Life. Four studies [9-11, 19] involving 202 patients evaluated QoL. Two studies used PDQ-8 to evaluate QoL, and the other 2 studies used PDQ-39 scale. The results indicated that yoga yielded significant improvement in terms of QoL of PD patients (SMD $=-0.54 ; 95 \% \mathrm{CI},-0.97$ to -0.11 ; $P=0.01)$. No substantial heterogeneity was detected among the studies $\left(I^{2}=35 \% ; P=0.20\right)$ (Figure 9).

\section{Discussion}

This meta-analysis was aimed at systematically evaluating the effects of yoga on patients with mild to moderate PD based on 10 eligible RCTs, including motor symptoms, nonmotor symptoms, and QoL. As we all know, exercise is an integral part of PD management, which has been shown to positively affect cognition, mood impairments, mobility, and balance and to slow the progression of PD $[24,25]$. However, traditional aerobic or resistance-based exercises require safety monitoring, and some workout need exercise equipment. Yoga is generally used by the public as a way of exercise that promotes health and wellness. Through combining sitting and standing postures with breathing and meditation techniques, yoga has proven to be beneficial to health [26]. Due to its gentle approach, yoga has shown promise as an intervention that can be appropriate for PD patients who may not be able to participate in strenuous or intensive exercise [21]. Our meta-analysis suggested that yoga might have beneficial effects on promoting motor status, functional mobility, balance function, anxiety, depression, and QoL in PD patients.

Few studies have investigated the influence of yoga on motor status of PD patients, and the results of this study showed a significant improvement in UPDRS-III scores, but the heterogeneity among the articles was high. Random-effects model was adopted, and sensitivity analysis was conducted to explore the source of heterogeneity. By eliminating individual study one by one, the decrease of $I^{2}$ to $0 \%$ was observed when the article conducted by $\mathrm{Ni}$ et al. [9] was removed, which meant that the heterogeneity between articles disappeared. Subgroup analysis showed that the average age of patients in the yoga group was over 70 years old in the study by $\mathrm{Ni}$ et al., while PD patients performing yoga exercise in other studies had an average age below 70 years old (Figure 4). Therefore, elderly age may be associated with the effect of yoga on motor status in PD. Studies have suggested that stretching exercises and prolonged physical postures in yoga can lengthen major muscle groups and activate the stretch receptors in muscles, ligaments, and joints, thereby improving physical strength and flexibility [27, 28]. The practice of yoga stretching posture could help to fully exercise the muscle strength of erector spinae, gluteus maximus, and rectus abdominis, increasing the stretching distance of the body, expanding the range of joint activities, and enhancing the flexibility of the lumbar, knee, and ankle joints [29]. Based on the abovementioned results, yoga can make the limb and joint activities of the individuals with PD more flexible, then reduce bradykinesia and rigidity, 


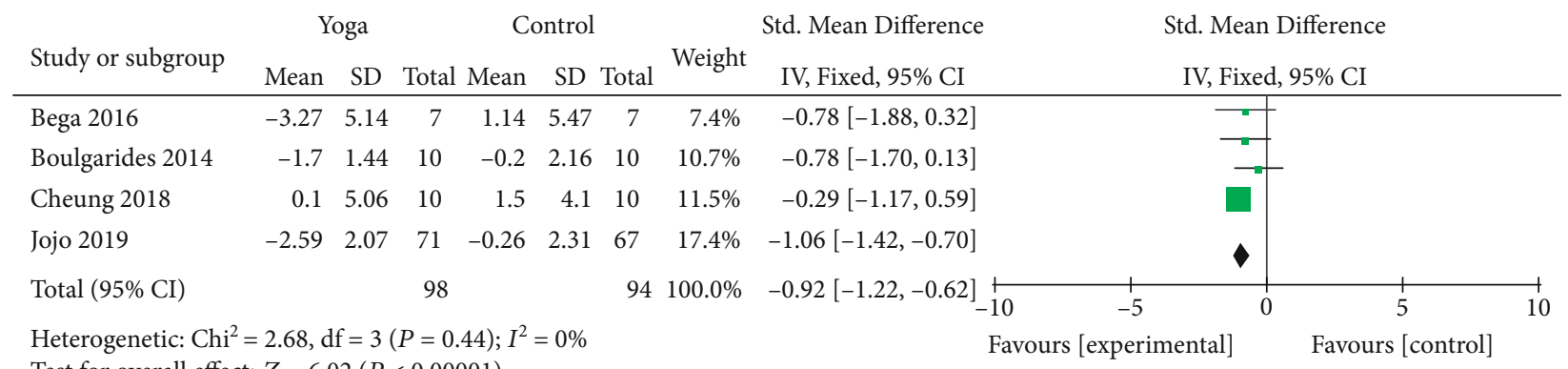

FIgURE 8: Forest plot from the meta-analysis of yoga on depression.

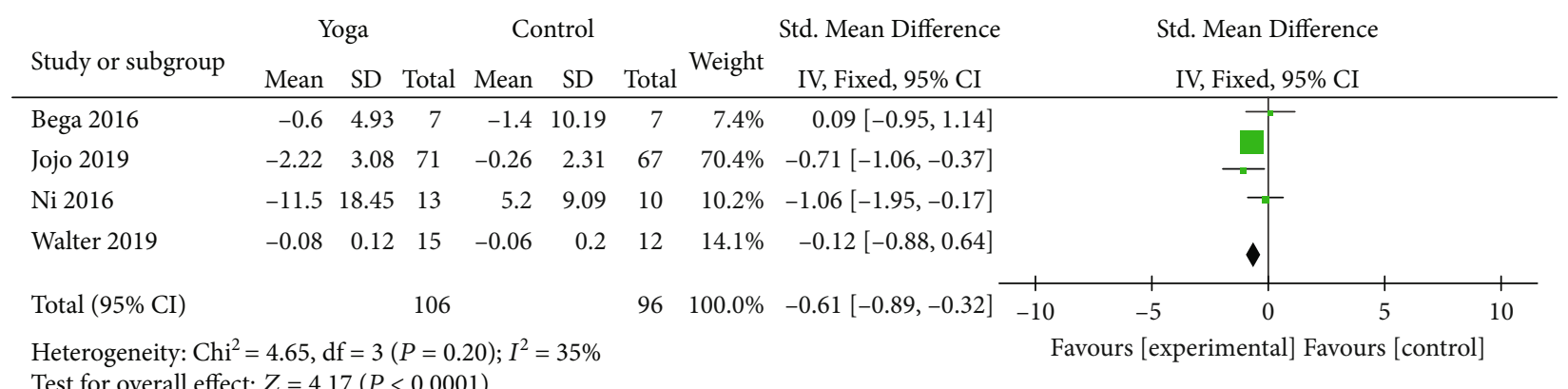

Figure 9: Forest plot from the meta-analysis of yoga on quality of life.

and increase the muscle strength and power. Moreover, yoga practice has demonstrated the greatest effect in improving motor symptoms in terms of UPDRS-III. These results were consistent with our findings.

Our meta-analysis also indicated that yoga had a significant effect on the improvement of TUG and balance function in PD. Patients with PD have an increased risk of falling due to decreased balance, reduced muscle strength, and freezing gait. The fear of falling (FOF) refers to decreased selfefficacy or low self-confidence to avoid falling during activities [30]. Studies have revealed that yoga could significantly improve the sensitivity of muscle proprioception, the stability of vestibular system, and the comprehensive analysis ability of cerebral cortex of PD patients by altering the fast and slow rhythms [31], so yoga intervention was shown to be successful in reducing the levels of FOF in individuals with PD. Nevertheless, yoga was considered as an alternative therapy, and there was evidence that balance training in yoga could make patients with PD more stable, straighter, and better at switching and anticipating movements. Many yoga postures challenge balance by placing the body in an unstable position, which in turn cannot be maintained without appropriate muscular activation to stabilize the joints [13].

Anxiety is a common nonmotor symptom that can affect up to $55 \%$ of patients with PD [32]. Previous studies have mentioned the benefits of yoga on anxiety, whereas the study by Myers et al. [13] showed that there was no significant decrease in BAI in the yoga group. Therefore, a more detailed statistical analysis was conducted, and the pooled results demonstrated that yoga could significantly ameliorate the symptoms of anxiety in patients with PD. Some studies have found that depression has a negative impact on energy levels, concentrating and decision-making, sleep, and perceived quality of life. Moreover, motor and cognitive competence were also negatively affected by depression in those with PD $[33,34]$. A statistically significant effect on depressive symptoms in patients with PD was observed in our metaanalysis. There was evidence that yoga exercise could correct underactive parasympathetic system and GABAergic system through vagal nerve stimulation, thereby reducing the allostatic load in the stress response system, which in turn might affect the brain's interpretation and response to internal stress and might enhance stress resilience $[35,36]$. Yoga has also been shown to decrease cortisol level and increase GABAergic activity, thereby improving patient mood and reducing anxiety $[37,38]$. The reduction of anxiety and depressive symptoms in the yoga group showed significant differences. However, due to the limitations in the number of articles and sample size, further studies were warranted to strengthen and consolidate these evidences.

Some evidences showed that the QoL of patients with PD was affected by functional impairment and nonmotor symptoms, which had a serious negative consequence for patients with PD. Our meta-analysis suggested that yoga significantly improved QoL of PD patients. In short, yoga could improve motor symptoms, reduce the emotions of anxiety and depression, increase QoL, and promote the development of physical and mental health of PD patients.

One of the included articles [11] reported adverse events. Both the yoga group and the control group demonstrated transient mild knee pain, but they required no medical attention. None of the included articles reported serious adverse events.

However, our study had some limitations, which were as follows: firstly, the loss of research protocol and trial registration information in most of the articles could introduce reporting bias. Publication bias might exist because funnel 


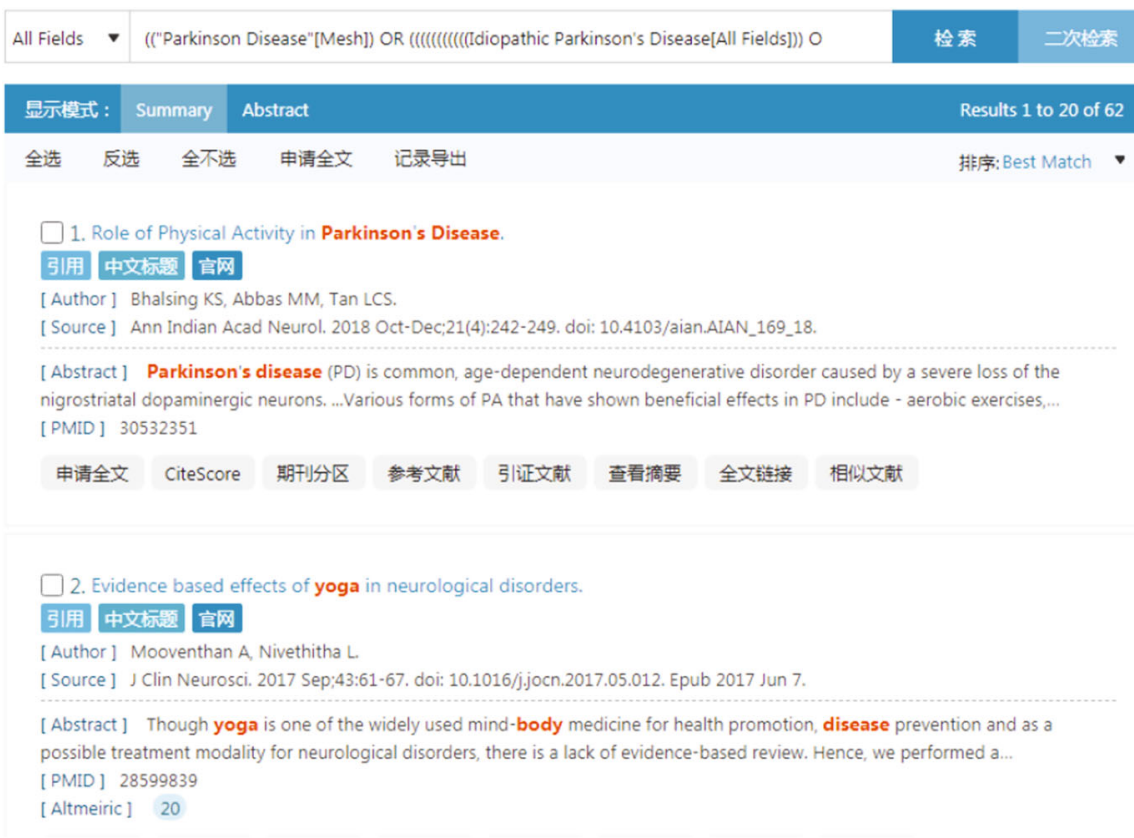

Figure 10: Results of literature search in PubMed database.

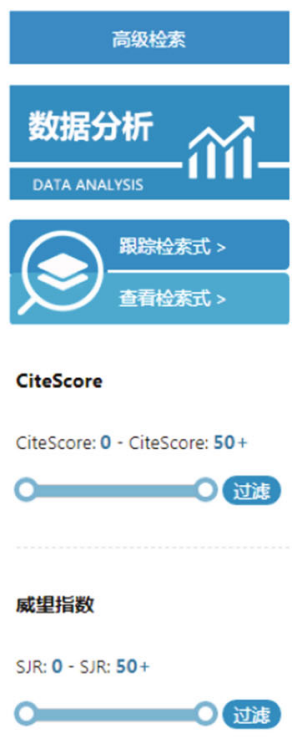

中科院分区过滤 plots could not be evaluated due to inclusion of small number of studies. Secondly, due to the inclusion of fewer studies, the sample size of our meta-analysis remained small, and some meaningful outcome measures were not analyzed in this study, such as Giladi Freezing of Gait Scale (FOG), 10Minute Walk test, and Montreal Cognitive Assessment (MoCA). Future studies should delve into these aspects to find out more benefits of yoga in the treatment of PD. Thirdly, most of the studies we included did not compare yoga with other complementary approaches such as Tai Chi, Tango, and Qi Gong. Whether yoga had a greater advantage than other exercises, whether it needed to be combined with other exercises, and what is the optimal amount of yoga exercise, all these needed further study. Next, we plan to make a profound study on the differences among yoga, Tai Chi, Tango, Qi Gong, and other traditional treatments. These limitations might affect the reliability of our results; therefore, our results should be interpreted with caution.

\section{Conclusion}

Our pooled results indicated that yoga might significantly improve the motor status, balance, functional mobility, and quality of life of individuals with PD and might reduce their anxiety and depression. Due to several limitations in this meta-analysis, well-designed studies with larger sample size and additional detailed outcomes were necessary to obtain clear conclusions of yoga on PD.

\section{Appendix}

\section{Search strategy}

\#1 (Parkinson Disease) OR (Idiopathic Parkinson's Disease) OR (Lewy Body Parkinson's Disease) OR (Parkinson's Dis- ease, Idiopathic) OR (Parkinson's Disease, Lewy Body) OR (Parkinson Disease, Idiopathic) OR (Parkinson's Disease) OR (Idiopathic Parkinson Disease) OR (Lewy Body Parkinson Disease) OR (Primary Parkinsonism) OR (Parkinsonism, Primary) OR (Paralysis Agitans) (Figure 10)

$$
\begin{aligned}
& \text { \#2 (Yoga) } \\
& \text { \#3 \#1 AND \#2 }
\end{aligned}
$$

\#4 (randomized controlled trial) OR (controlled clinical trial) OR (randomized) OR (placebo) OR (drug therapy) OR (randomly) OR (trial) OR (groups) NOT (animals) NOT (humans)

\#5 \#3 AND \#4

Pubmed:

(((Parkinson Disease) OR ${ }^{(()((()(((I d i o p a t h i c ~ P a r k i n-~}$ son's Disease[All Fields])) OR (Lewy Body Parkinson's Disease)) OR (Parkinson's Disease, Idiopathic)) OR (Parkinson's Disease, Lewy Body)) OR (Parkinson Disease, Idiopathic)) OR (Parkinson's Disease)) OR (Idiopathic Parkinson Disease)) OR (Lewy Body Parkinson Disease)) OR (Primary Parkinsonism)) OR (Parkinsonism, Primary)) OR (Paralysis Agitans)) AND (Yoga) AND $(((()((($ randomized controlled trial)) OR (controlled clinical trial)) OR (randomized)) OR (placebo)) OR (drug therapy)) OR (randomly)) OR (trial)) OR (groups)) NOT ((animals)) NOT (humans).

\section{Conflicts of Interest}

No conflict of interest has been declared by the authors.

\section{Authors' Contributions}

Mengke Ban takes charge of researching, data curation and analysis, writing, and revising. Xuejing Yue plays an important guiding role in literature search, quality assessment, and paper revision. Pengyu Dou has made contributions to 
data extraction. Ping Zhang is mainly responsible for decision-making research direction, deciding on the final manuscript, and providing financial support.

\section{Acknowledgments}

This study was funded by the (1) National Science Foundation of China (No. 81471349) and (2) Henan Key Laboratory of Neurorestoratology (HNSJXF-2018-007).

\section{References}

[1] A. Elbaz, L. Carcaillon, S. Kab, and F. Moisan, "Epidemiology of Parkinson's disease," Revue Neurologique, vol. 172, no. 1, pp. 14-26, 2016.

[2] J. Jankovic, "Parkinson's disease: clinical features and diagnosis," Journal of Neurology, Neurosurgery, and Psychiatry, vol. 79, no. 4, pp. 368-376, 2008.

[3] K. R. Chaudhuri, D. G. Healy, and A. H. Schapira, "Non-motor symptoms of Parkinson's disease: diagnosis and management," The Lancet Neurology, vol. 5, no. 3, pp. 235-245, 2006.

[4] K. Ray Chaudhuri, W. Poewe, and D. Brooks, "Motor and nonmotor complications of levodopa: phenomenology, risk factors, and imaging features," Movement Disorders, vol. 33, no. 6, pp. 909-919, 2018.

[5] H. A. Tindle, R. B. Davis, R. S. Phillips, and D. M. Eisenberg, "Trends in use of complementary and alternative medicine by US adults: 1997-2002," Alternative Therapies in Health and Medicine, vol. 11, no. 1, pp. 42-49, 2005.

[6] E. Green, A. Huynh, L. Broussard et al., "Systematic review of yoga and balance: effect on adults with neuromuscular impairment," American journal of occupational therapy, vol. 73, no. $1,2019$.

[7] X. Jin, L. Wang, S. Liu, L. Zhu, P. D. Loprinzi, and X. Fan, “The impact of mind-body exercises on motor function, depressive symptoms, and quality of life in Parkinson's disease: a systematic review and meta-analysis," International journal of environmental research and public health, vol. 17, no. 1, p. 31, 2020.

[8] L. M. Deuel and L. C. Seeberger, "Complementary therapies in Parkinson disease: a review of acupuncture, Tai Chi, Qi Gong, yoga, and cannabis," Neurotherapeutics, vol. 17, no. 4, pp. 1434-1455, 2020.

[9] M. Ni, J. F. Signorile, K. Mooney et al., "Comparative effect of power training and high-speed yoga on motor function in older patients with Parkinson disease," Archives of physical medicine and rehabilitation, vol. 97, no. 3, pp. 345-354.e15, 2016.

[10] D. Bega and J. Stein, "Yoga versus resistance training in mild to moderate severity Parkinson's disease: a 12-week pilot study," Journal of yoga \& physical therapy, vol. 6, no. 1, 2016.

[11] J. Y. Y. Kwok, J. C. Y. Kwan, M. Auyeung et al., "Effects of mindfulness yoga vs stretching and resistance training exercises on anxiety and depression for people with Parkinson disease: a randomized clinical trial," JAMA Neurology, vol. 76, no. 7, pp. 755-763, 2019.

[12] L. K. Boulgarides, E. Barakatt, and B. Coleman-Salgado, "Measuring the effect of an eight-week adaptive yoga program on the physical and psychological status of individuals with Parkinson's Disease. A Pilot Study," The International Journal of Yoga Therapy, vol. 24, no. 1, pp. 31-41, 2014.
[13] P. S. Myers, E. C. Harrison, K. S. Rawson et al., "Yoga improves balance and low-back pain, but not anxiety, in people with Parkinson's disease," International journal of yoga therapy, vol. 30, no. 1, pp. 41-48, 2020.

[14] N. K. Sharma, K. Robbins, K. Wagner, and Y. M. Colgrove, “A randomized controlled pilot study of the therapeutic effects of yoga in people with Parkinson's disease," International journal of yoga, vol. 8, no. 1, pp. 74-79, 2015.

[15] J. Y. Y. Kwok, J. C. Y. Kwan, M. Auyeung, V. C. T. Mok, and H. Y. L. Chan, "The effects of yoga versus stretching and resistance training exercises on psychological distress for people with mild-to-moderate Parkinson's disease: study protocol for a randomized controlled trial," Trials, vol. 18, no. 1, p. 509, 2017.

[16] M. Ni, K. Mooney, and J. F. Signorile, "Controlled pilot study of the effects of power yoga in Parkinson's disease," Complementary Therapies in Medicine, vol. 25, pp. 126-131, 2016.

[17] B. L. Hawkins, M. Van Puymbroeck, A. Walter et al., "Perceived activities and participation outcomes of a yoga intervention for individuals with Parkinson's disease: a mixed methods study," International journal of yoga therapy, vol. 28, no. 1, pp. 51-61, 2018.

[18] M. van Puymbroeck, A. A. Walter, B. L. Hawkins et al., "Functional Improvements in Parkinson's Disease Following a Randomized Trial of Yoga," Evidence-based Complementary and Alternative Medicine: Ecam, vol. 2018, article 8516351, pp. 18, 2018.

[19] A. A. Walter, E. V. Adams, M. Van Puymbroeck et al., "Changes in nonmotor symptoms following an 8-week yoga intervention for people with Parkinson's disease," International journal of yoga therapy, vol. 29, no. 1, pp. 91-99, 2019.

[20] N. P. Cherup, K. L. Strand, L. Lucchi, S. V. Wooten, C. Luca, and J. F. Signorile, "Yoga meditation enhances proprioception and balance in individuals diagnosed with Parkinson's disease," Perceptual and Motor Skills, vol. 128, no. 1, pp. 304323, 2020.

[21] C. Cheung, R. Bhimani, J. F. Wyman et al., "Effects of yoga on oxidative stress, motor function, and non-motor symptoms in Parkinson's disease: a pilot randomized controlled trial," Pilot and feasibility studies, vol. 4, no. 1, p. 162, 2018.

[22] Y. Colgrove and N. Sharma, "Effect of yoga on motor function in people with Parkinson's disease: a randomized, controlled pilot study," Journal of Yoga \& Physical Therapy, vol. 2, no. 2, 2012.

[23] A. Khuzema, A. Brammatha, and V. Arul Selvan, "Effect of home-based Tai Chi, Yoga or conventional balance exercise on functional balance and mobility among persons with idiopathic Parkinson's disease: an experimental study," Hong Kong physiotherapy journal: official publication of the Hong Kong Physiotherapy Association Limited $=W u$ li chih liao, vol. 40, no. 1, pp. 39-49, 2020.

[24] K. E. Cruise, R. S. Bucks, A. M. Loftus, R. U. Newton, R. Pegoraro, and M. G. Thomas, "Exercise and Parkinson's: benefits for cognition and quality of life," Acta Neurologica Scandinavica, vol. 123, no. 1, pp. 13-19, 2011.

[25] O. Oguh, A. Eisenstein, M. Kwasny, and T. Simuni, "Back to the basics: regular exercise matters in parkinson's disease: results from the National Parkinson Foundation QII registry study," Parkinsonism \& Related Disorders, vol. 20, no. 11, pp. 1221-1225, 2014.

[26] K. J. Sherman, "Guidelines for developing yoga interventions for randomized trials," Evidence-based Complementary and Alternative Medicine: Ecam, vol. 2012, article 143271, pp. 1$16,2012$. 
[27] F. M. Luskin, K. A. Newell, M. Griffith et al., "A review of mind/body therapies in the treatment of musculoskeletal disorders with implications for the elderly," Alternative Therapies in Health and Medicine, vol. 6, no. 2, pp. 46-56, 2000.

[28] M. D. Tran, R. G. Holly, J. Lashbrook, and E. A. Amsterdam, "Effects of hatha yoga practice on the health-related aspects of physical fitness," Preventive Cardiology, vol. 4, no. 4, pp. 165-170, 2001.

[29] “管细红, 朱晓钢, 刘建民, 刘志强, and 翟绍征, 瑜伽运动对 早期帕金森病人平衡能力及害怕跌倒的影响. 护理研究, ” vol. 31, pp. 1274-1276, 2017.

[30] M. E. Tinetti and L. Powell, "4 Fear of falling and low self-efficacy: a Cause of dependence in elderly persons," Journal of gerontology, vol. 48, pp. 35-38, 1993.

[31] “陈龙, 健身功法对老年人平衡能力的影响研究. 体育文化 导刊,” pp. 99-101, 2015.

[32] M. P. Broen, N. E. Narayen, M. L. Kuijf, N. N. Dissanayaka, and A. F. Leentjens, "Prevalence of anxiety in Parkinson's disease: a systematic review and meta-analysis," Movement disorders: official journal of the Movement Disorder Society, vol. 31, no. 8, pp. 1125-1133, 2016.

[33] J. S. Reijnders, U. Ehrt, W. E. Weber, D. Aarsland, and A. F. Leentjens, "A systematic review of prevalence studies of depression in Parkinson's disease," Movement disorders: official journal of the Movement Disorder Society, vol. 23, no. 2, pp. 183-189, 2008, quiz 313.

[34] A. H. Farabaugh, J. J. Locascio, L. Yap et al., "Pattern of depressive symptoms in Parkinson's disease," Psychosomatics, vol. 50, no. 5, pp. 448-454, 2009.

[35] R. P. Brown and P. L. Gerbarg, "Sudarshan Kriya yogic breathing in the treatment of stress, anxiety, and depression: part Ineurophysiologic model," Journal of alternative and complementary medicine (New York, N.Y.), vol. 11, no. 1, pp. 189201, 2005.

[36] C. C. Streeter, P. L. Gerbarg, R. B. Saper, D. A. Ciraulo, and R. P. Brown, "Effects of yoga on the autonomic nervous system, gamma-aminobutyric-acid, and allostasis in epilepsy, depression, and post-traumatic stress disorder," Medical Hypotheses, vol. 78, no. 5, pp. 571-579, 2012.

[37] T. Kamei, Y. Toriumi, H. Kimura, H. Kumano, S. Ohno, and K. Kimura, "Decrease in serum cortisol during yoga exercise is correlated with alpha wave activation," Perceptual and Motor Skills, vol. 90, no. 3, pp. 1027-1032, 2000.

[38] C. C. Streeter, T. H. Whitfield, L. Owen et al., "Effects of yoga versus walking on mood, anxiety, and brain GABA levels: a randomized controlled MRS study," Journal of alternative and complementary medicine (New York, N.Y.), vol. 16, no. 11, pp. 1145-1152, 2010. 